\title{
IMMEDIATE COMMUNICATION \\ Patient-specific models of microglia-mediated engulfment of synapses and neural progenitors
}

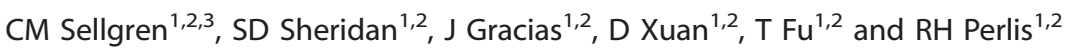

\begin{abstract}
Engulfment of synapses and neural progenitor cells (NPCs) by microglia is critical for the development and maintenance of proper brain circuitry, and has been implicated in neurodevelopmental as well as neurodegenerative disease etiology. We have developed and validated models of these mechanisms by reprogramming microglia-like cells from peripheral blood mononuclear cells, and combining them with NPCs and neurons derived from induced pluripotent stem cells to create patient-specific cellular models of complement-dependent synaptic pruning and elimination of NPCs. The resulting microglia-like cells express appropriate markers and function as primary human microglia, while patient-matched macrophages differ markedly. As a demonstration of diseaserelevant application, we studied the role of C4, recently implicated in schizophrenia, in engulfment of synaptic structures by human microglia. The ability to create complete patient-specific cellular models of critical microglial functions utilizing samples taken during a single clinical visit will extend the ability to model central nervous system disease while facilitating high-throughput screening.
\end{abstract}

Molecular Psychiatry (2017) 22, 170-177; doi:10.1038/mp.2016.220; published online 13 December 2016

\section{INTRODUCTION}

In light of their importance in normal development of the mammalian central nervous system, microglia have been proposed to contribute to the pathogenesis of neurodevelopmental and/or neurodegenerative diseases as well. ${ }^{1}$ Large-scale functional studies of human microglia in disease have been hampered by difficulties in obtaining and studying live human cells, particularly from affected individuals. Although human microglia have been isolated from biopsy ${ }^{2}$ as well as autopsy ${ }^{3}$ samples, these approaches are not suitable for adequately powered statistical comparisons of healthy individuals with affected ones, or for highthroughput drug screening. Thus far, evidence for microgliamediated elimination of synapses, a process commonly referred to as synaptic pruning, as well as microglia regulation of the neural progenitor cell (NPC) pool by engulfment of live and apoptotic NPCs, is based on rodent studies. ${ }^{4-9}$ Similar, functional diseaseorientated studies are limited to murine models such as the discovery of impaired engulfment of NPCs by murine Mecp $2^{-/-}$ microglia (Rett syndrome), ${ }^{10}$ complement-dependent synapse elimination by microglia mimicking early synaptic loss in a mouse model of Alzheimer's disease ${ }^{11}$ or reduced synaptic pruning by $\mathrm{Grn}^{-1-}$ microglia (familial frontotemporal lobar degeneration). ${ }^{12}$ Unfortunately, rodent microglia display biochemical and pharmacological differences from their human counterparts ${ }^{13}$ and are limited in their ability to model complex genetic diseases. Conversely, the ability to reprogram easily-accessible human somatic cells in a clinical setting would provide a unique opportunity for patient-specific high-throughput modeling of microglia dysfunction. Therefore, with a focus on recapitulating features of the human central nervous system microenvironment, we set out to (1) generate human microglia-like cells from human somatic cells and validate them against primary cells, (2) develop an in vitro model system for microglial elimination of synapses and NPCs from human primary and reprogrammed cells and (3) demonstrate psychiatric disease-relevant application.

\section{MATERIALS AND METHODS}

Ethical statement

The study was approved by the Partners Institutional Review Board. Informed consent was obtained from all participants.

\section{Preparation of peripheral blood mononuclear cells from whole blood}

Blood was collected into acid citrate dextrose solution using vacutainer tubes. The blood was then transferred into mononuclear cell preparation tubes (Becton-Dickson, Franklin Lakes, NJ, USA; \#362761), which were first voided of the anticoagulant. The tubes were spun in room temperature at $1700 \mathrm{~g}$ for 30-40 min. The plasma was then aspirated off and the peripheral blood mononuclear cells (PBMCs) were lifted with a pipette and recovered into a $15 \mathrm{ml}$ conical tube. The tube was filled to top with phosphate-buffered saline (PBS) and spun in room temperature at $380 \mathrm{~g}$ for $15 \mathrm{~min}$. Once the tubes had spun, the supernatant was poured out and the solution was resuspended in $10 \mathrm{ml}$ PBS and spun for $10 \mathrm{~min}$ at $280 \mathrm{~g}$. After that spin, the supernatant was poured out and the pellet was resuspended into $2 \mathrm{ml}$ fetal bovine serum (FBS) with $10 \%$ dimethyl sulfoxide and aliquoted into two $1 \mathrm{ml}$ Nunc CryoTubes vials (Thermo Fisher Scientific, Waltham, MA, USA). The vials were gradually brought

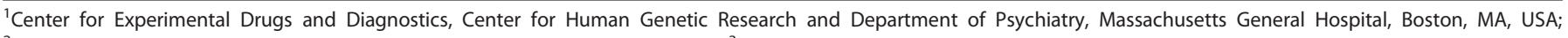

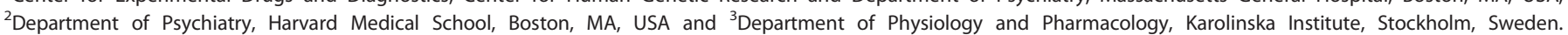

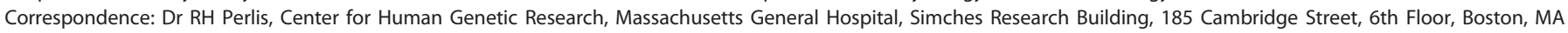
02114, USA. 
down to temperature, first on wet ice, then into $-80^{\circ} \mathrm{C}$ and finally moved into liquid nitrogen storage.

Generation of induced microglia-like cells from PBMCs

PBMC samples from the liquid nitrogen tanks were quick thawed in a $37^{\circ} \mathrm{C}$ water bath for approximately $2 \mathrm{~min}$. The buffy coat was then pipetted into $9 \mathrm{ml}$ of pre warmed RPMI-1640 (Sigma, St. Louis, MO, USA; \#R0883-500 MI) with 10\% heat-inactivated low-endotoxin FBS (Rockland Immunochemicals; \#FBS-01-0100) and $1 \%$ penicillin-streptomycin $(\mathrm{P} / \mathrm{S})$. The tube was then centrifuged at $300 \mathrm{~g}$ for $5 \mathrm{~min}$ with the deceleration brake off. The supernatant was aspirated completely and the pellet was resuspended in the appropriate volume of RPMI-1640 with $10 \%$ FBS and $1 \% \mathrm{P} / \mathrm{S}$ medium. Cells were plated at a density of 500,000 cells per well on 24-well plates coated with Geltrex (Thermo Fisher Scientific). After $24 \mathrm{~h}$ of incubation, the media was carefully aspirated with a $200 \mu \mathrm{l}$ pipette. Media was replaced with RPMI-1640 Glutamax (Life Technologies, Carlsbad, CA, USA) supplemented with $1 \% \mathrm{P} / \mathrm{S}$ and $0.1 \mu \mathrm{g} \mathrm{ml}^{-1}$ of interleukin (IL)-34 (R\&D Systems, Minneapolis, MN, USA) and $0.01 \mathrm{\mu g} \mathrm{ml}^{-1}$ of granulocyte macrophage colony-stimulating factor (R\&D Systems; \#215-GM-010/CF). After 9 days the plates were washed thoroughly to remove any unbound cells and fresh medium was added. Cells were then harvested or used for functional assays the following day.

Generation of monocyte-derived macrophages from PBMCs

Macrophages were generated from buffy coats as described above but with some exceptions. First, we used non-coated 24well plates. At day 1, medium was also changed to RPMI-1640 Glutamax supplemented with the addition of $10 \%$ FBS and granulocyte macrophage colony-stimulating factor $\left(0.01 \mathrm{\mu g} \mathrm{ml}^{-1}\right)$ and medium change was performed every third day thereafter.

Microglia purification from post-mortem human brain

Hippocampal post-mortem brain samples (age 70-80, Caucasian, both male and female) were procured 9-12 h post mortem by the National Disease Research Interchange (NDRI) with support from $\mathrm{NIH}$ grant 2 U42 OD011158. Dissected brain sections were weighed and cut into fine pieces with a scalpel blade and homogenized into $10 \mathrm{ml}$ of Dulbecco's modified Eagle's medium (DMEM)/F12 (Gibco, Waltham, MA, USA) with 1\% B27 (Gibco) using a $15 \mathrm{ml}$ Dounce Tissue Grinder (Wheaton, Millville, NJ, USA; \#1526784). The suspension was strained through a 70-micron filter (Becton-Dickson) and the cells were then centrifuged at $160 \mathrm{~g}$ for $10 \mathrm{~min}$ at $4{ }^{\circ} \mathrm{C}$. The supernatant was aspirated and the cell pellet was resuspended in media that contained DMEM/F12 with $1 \%$ B27, $1 \%$ Glutamax, $1 \% \mathrm{P} / \mathrm{S}$, fibroblast growth factor $2\left(40 \mathrm{ng} \mathrm{ml}^{-1}\right)$, epidermal growth factor $\left(40 \mathrm{ng} \mathrm{ml}^{-1}\right), 1 \%$ Amphotericin B and heparin $\left(2 \mu \mathrm{g} \mathrm{ml}^{-1}\right)$. The cell suspension was plated in a T75 tissue culture flask and incubated at $37^{\circ} \mathrm{C}$. After $24 \mathrm{~h}$, the flask was firmly tapped to remove non-adherent cells. After two washes the media was replaced by DMEM/F12 with 10\% FBS, 1\% P/S and 1\% Amphotericin B. Medium change was performed every third day and at day 9 we serum starved the cells for $24 \mathrm{~h}$. Cells were then harvested at day 10. Between 90 and $95 \%$ of the isolated cells stained positive for CX3CR1 (Supplementary Figure S1) and CD11b.

\section{Human Lenti-SV40 immortalized microglia cell line}

Cryopreserved and Lenti simian virus-40 (SV40) immortalized human microglia cells, derived from human fetal brain tissue (>99\% purity), were purchased and cultured according to the manufacturer's instructions (Innoprot, Derio, Bizkaia, Spain). Cells had been tested for the microglial markers IBA1, CD68, TREM2 and CD11b, and were negative for HIV-1, HBV, HCV, mycoplasma, bacteria, yeast and fungi. Briefly, microglia were cultured on collagen-coated 24-well plates (Corning, Corning, NY, USA) at a density of 10,000 cells per $\mathrm{cm}^{2}$ in a complete medium provided by the manufacturer that contained 20\% FBS and 5\% microglia growth supplement (\#P60116). All used cells were in passage 3 or 4 after immortalization.

\section{Human fetal primary microglia}

Cryopreserved primary microglia cells, originally derived from human fetal brain tissue, were purchased and cultured according to the manufacturer's instructions $(3 \mathrm{H}$ Biomedical, Uppsala, Sweden). Briefly, microglia were cultured on poly-L-lysine-coated 24 -well plates at a density of 10,000 cells per $\mathrm{cm}^{2}$ in DMEM/F12 medium supplemented with $0.01 \mathrm{\mu g} \mathrm{ml}^{-1}$ of granulocyte macrophage colony-stimulating factor and $1 \% \mathrm{P} / \mathrm{S}$. Cells were tested for the markers CD45, CD14 and CD68, and were negative for HIV, $\mathrm{HBV}, \mathrm{HCV}$, mycoplasma, bacteria, yeast and fungi.

\section{Biopsy and fibroblast derivation}

A dermal biopsy was obtained by a physician investigator. Following subcutaneous injection of lidocaine $1 \%$, a 3.0 or $4.0 \mathrm{~mm}$ punch tool was used to obtain a sample from the nondominant forearm. Biopsies where then collected into a transport medium of DMEM (Gibco) with $1 \%$ penicillin-streptomycinglutamine (Corning Life Sciences, Tewksbury, MA, USA). Upon receipt of samples, they were washed twice in PBS with $10 \%$ penicillin-streptomycin-glutamine. Two $60-\mathrm{mm}$ tissue culturetreated plates were used, one to cut up the biopsy and one to plate the pieces of biopsy. A scalpel was used to cut the biopsy into the smallest pieces possible and then to grid the dish where the pieces of biopsy were plated dermis side down. The dish was placed in an incubator at $37^{\circ}$ for $15 \mathrm{~min}$ in order for the skin to adhere to the dish. Medium of DMEM with $10 \%$ FBS and $1 \%$ penicillin-streptomycin-glutamine was finally gently added over the pieces of skin.

Induced pluripotent stem cell reprogramming

Human fibroblasts were reprogrammed and the resulting iPSC colonies stabilized and expanded under xeno-free conditions by Stemiotics (San Diego, CA, USA). Each line was plated to six-well plates without feeders at three different plating densities and then subjected to messenger RNA reprogramming. Colonies were bulkpassaged from the most productive well to establish passage 1 (P1) iPSC cultures on rLaminin-521 (BioLamina, Sundbyberg, Sweden) in Nutristem XF media (Biological Industries, Kibbutz Beit-Haemek, Israel) and expanded in the same culture system until at least passage 3 before being frozen down for storage.

Neural progenitor derivation and neural differentiation

iPSCs were cultured feeder-free in E8 medium (Gibco) on Geltrexcoated six-well plates and passaged using $50 \mathrm{~mm}$ EDTA and trituration with ROCK inhibitor $(10 \mathrm{~mm}$ Thiazovivin; Stemgent, Lexington, MA, USA). iPSCs were purified using magnetic-activated cell sorting with Tra-1-60 microbeads (Miltenyi Biotec, Bergisch Gladbach, Germany) on LS columns as described by vendor. Sorted cells were further cultured until appropriate confluence before neural induction (10-20\%). Neural induction was initiated by media replacement to Neurobasal media (Gibco) supplemented with $1 \times$ Neural Induction Supplement (NIS media) (Gibco). Cell were cultured to confluence and further expanded in expansion media (NEM-50\% Neurobasal, 50\% advanced DMEM/F12 with $1 \times$ Neural Induction Supplement; Gibco) passaged by accutase digestion. Putative NPCs were further purified by PSANCAM magnetic-activated cell sorting (Miltenyi Biotec) for further culture and cryopreservation in NEM supplemented with $10 \%$ dimethyl sulfoxide. Neural differentiation from NPCs for the purpose of generating synaptosomes was initiated by seeding onto Geltrex-coated T75 flask at a cell density of approximately 
40,000 cells per $\mathrm{cm}^{2}$ in NEM. Media was switched the following day to basal differentiation media containing 70\% DMEM/30\% Ham's F12 supplemented with $1 \times$ B27 (Gibco) and $1 \times$ PennStrep (Gibco). Media was then completely changed every 3-4 days. Twenty-four hours before harvesting medium was changed to serum-free astrocyte-conditioned medium (ACM; ScienCell, Carlsbad, CA, USA). Cells were then harvested by scraping at approximately 28 days.

\section{Purification and labeling of NPCs and synaptosomes}

Synaptosomes were prepared from NPC-derived neural cultures and adult human brain post-mortem samples using Syn-PER Synaptic Protein Extraction Reagent (Thermo Fisher Scientific). For neural cultures, the included primary cultured neurons protocol was followed with $2 \mathrm{ml}$ Syn-Per reagent per T75 culture flask. Briefly, after aspirating cell medium (here ACM), followed by two washes, the Syn-PER reagent is added. Cells are lifted off the plate by a cell scraper and the sample is centrifuged at $1200 \mathrm{~g}$ for $10 \mathrm{~min}$. The supernatant is then centrifuged at 15,000 $\mathrm{g}$ for $20 \mathrm{~min}$ and the pellet containing synaptosomes is resuspended in medium (here RPMI-1640) before used for assay. For brain samples, homogenization, digestion and purification was performed using $10 \mathrm{ml}$ of Syn-PER reagent per gram of tissue following the procedure for neural tissue on weighed dissected samples. Resulting synaptosomes were collected and labeled with an amine-reactive dye (pHrodo Red, SE; Thermo Scientific; \#P36600) according to data sheet instructions for labeling. This $\mathrm{pH}$-sensitive dye binds non-specifically to proteins and fluoresces bright red in the post-phagocytic phagolysosome compartments. The lack of fluorescence outside the cell then enables robust quantification of cell uptake and has previously been used to study microglial uptake of mouse synaptosomes. ${ }^{14}$ As previously described for other cell types, ${ }^{15}$ we also used pHrodo for labeling live NPCs. Cells were harvested by gentle scraping, spun at $300 \mathrm{~g}$ for $5 \mathrm{~min}$, and resuspended in freshly prepared $100 \mathrm{~mm}$ sodium bicarbonate solution $(\mathrm{pH}$ 8.5). The cell suspension was then incubated for $40 \mathrm{~min}$ in $0.01 \mathrm{mg} \mathrm{ml}^{-1} \mathrm{pHrodo}$ at room temperature and under protection from light (cell viability $>90 \%$ ).

\section{Western blot analysis}

Five micrograms (PSD-95, synapsin) or $20 \mu \mathrm{g}$ (C4) lysates were loaded onto BioRad's Criterion 4-12\% gel in repeat experiment. Proteins were transferred to PVDF membrane. For C4 experiments, membrane was blocked with 5\% milk TBST and probed with AntiC4 (1:5000; Abcam, Cambridge, UK; \#ab173577). Anti-Rabbit lgGHRP (1:1000; Cell Signaling Technology, Danvers, MA, USA; \#7074) was used for signal detection. For synapsin and PSD-95 detection membranes were blocked and probed with Anti-Synapsin (1:2000; Synaptic Systems, Goettingen, Germany; \#106-001) and AntiPSD-95 (1:5000; EMD Millipore, Billerica, MA, USA; \#MABN68), respectively. Anti-Mouse IgG-HRP (1:5000; Cell Signaling Technology; \#7076) was used for signal detection. Primary Anti- $\beta$-Actin (1:10,000; Sigma; \#A1978) and Anti-mouse IgG-HRP (1:5000; Cell Signaling Technology; \#7076) were employed for loading normalization based on $\beta$-actin expression.

\section{Immunocytochemistry}

Cells were fixed with $4 \%$ paraformaldehyde for $15 \mathrm{~min}$. Following fixation, they were washed three times with PBS. Primary antibodies were diluted in PBS with 1\% FBS as follows: CX3CR1 (Novus Biologicals; \#H00001524-B01P; 1:500), Anti-P2RY12 (Alomone Labs, Jerusalem, Israel; \#APR-020; 1:100), C4 (Abcam; \#173577; 1:250), CD11b (Abcam; \#133357; 1:250) and PSD-95 (EMD Millipore; \#MABN68; 1:500), IBA1 (Abcam ab15690; 1:200), TMEM119 (Abcam; ab185337; 1:200), PU.1/Spi1 (Abcam; ab88082; 1:200), MAP2 (Abcam; ab92434, 1:1000), SMI312 (Biolegend; 837904,
1:1000) and $\beta$ III-Tubulin (Millipore; MAB5564; 1:1500). Cells were incubated with primary antibodies for $2 \mathrm{~h}$ at room temperature or at $4{ }^{\circ} \mathrm{C}$ overnight. Cells were washed two times (10 min each) with $1 \%$ FBS in PBS followed by incubation with secondary antibodies (diluted 1:500 in PBS with 1\% FBS) and Hoechst (1:5000) for $1 \mathrm{~h}$ at room temperature in the dark. Cells were then washed twice with $1 \%$ FBS in PBS. Immunocytochemistry experiments were controlled with primary and secondary antibody controls.

\section{Nanostring nCounter gene and miRNA expression analysis}

Gene and miRNA expression levels were quantified using the Nanostring nCounter system (Nanostring Technologies, Seattle, WA, USA) with a custom panel of probes encompassing microglial identity (gene panel), NPC identity (gene panel) or the Human v3 nCounter miRNA Expression Assay panel of 800 miRNAs. Genes were selected based on previous data suggesting high enrichment in mouse or human microglia vs other immune cells, or in human microglia vs other brain cells. ${ }^{2,16}$ Total RNA was isolated using the Qiagen miRNeasy mini-kit per instructions including optional on-column DNasel treatment. RNA samples were quantitated using a Nanodrop 2000 UV-Vis Spectrophotometer (NanoDrop Technologies). Approximately 100ng of RNA was used for each Nanostring sample preparation as per included instructions. Output data was subject to quality and normalization control using Nanostring nSolver v2.5 software. miRNAs were selected for further analyses and clustering based on average of $>50$ counts across samples above in-chip background control. Log2-transformed expression levels were then used for hierarchical cluster analyses using median linking and correlation as distance metric. To assess the uncertainty in the hierarchical cluster analysis we applied bootstrap resampling (10,000 iterations) via the $\mathrm{R}$ package pvclust. For each suggested cluster pvclust provides two types of $P$-values: the Bootstrap Probability value from the ordinary bootstrap resampling and the Approximately Unbiased (AU) probability value from multiscale bootstrap resampling. The multiscale bootstrap resampling method was introduced to develop an approximately unbiased test, and therefore it provides better estimations of the probability values. ${ }^{17}$ The standard errors of AU P-values were also plotted against $A U P$-values with no indication of sampling error.

Real-time live cellimaging

Cells were imaged on 24-well plates using an IncuCyte ZOOM system (Essen Bioscience, Ann Arbor, MI, USA) incubated at $37^{\circ} \mathrm{C}$ and $5 \% \mathrm{CO}_{2}$. Four to nine images $(\times 20)$ per well were taken every $30 \mathrm{~min}$ for $5 \mathrm{~h}$ using the phase-contrast and red fluorescence mode. To validate that fluorescent pHrodo represented actual cell uptake, we used $1 \mathrm{~h}$ pre-treatment with Cytochalasin D (Biotrend, Ann Arbor, MI, USA; \#BS0056), an inhibitor of actin polymerization, at a concentration of $10 \mu \mathrm{m}$ as previously described. ${ }^{18}$ This reduced pHrodo-labeled uptake $>85 \%$. CD11b blocking experiments were performed by pre-treatment for 20 min using a functional anti-CD11b rat monoclonal lgG2b antibody of clone M1/70 (Abcam; \#ab180543; $6 \mathrm{\mu g} \mathrm{ml}^{-1}$ ) or a rat monoclonal lgG2b isotype control antibody (Abcam; \#ab154434; $6 \mu \mathrm{g} \mathrm{ml}^{-1}$ ). Quantification was performed using the IncuCyte ZOOM Software (2016A). Fluorescent pHrodo was separated from background by thresholding a top-Hat transform of the original image. All settings were kept constant between different conditions. As outcome measure we used total red object area $\left(\mu \mathrm{m}^{2}\right)$ per image divided on total number of cells per image. Before comparing uptake of pHrodo-labeled synaptosomes in microglia and monocyte-derived macrophages, we established the phagocytic competence of our generated monocyte-derived macrophages using pHrodo Red Zymosan Bioparticles according to data sheet instructions from the manufacturer (Life Technologies). This confirmed functional macrophages (Supplementary Figure S2). 
a

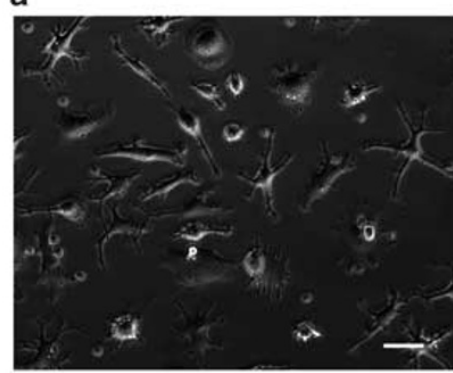

b

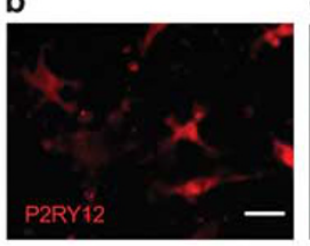

C

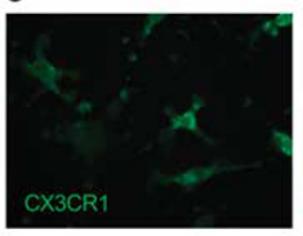

d

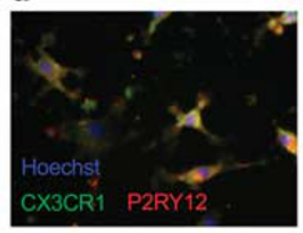

$\mathbf{f}$

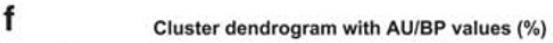

e
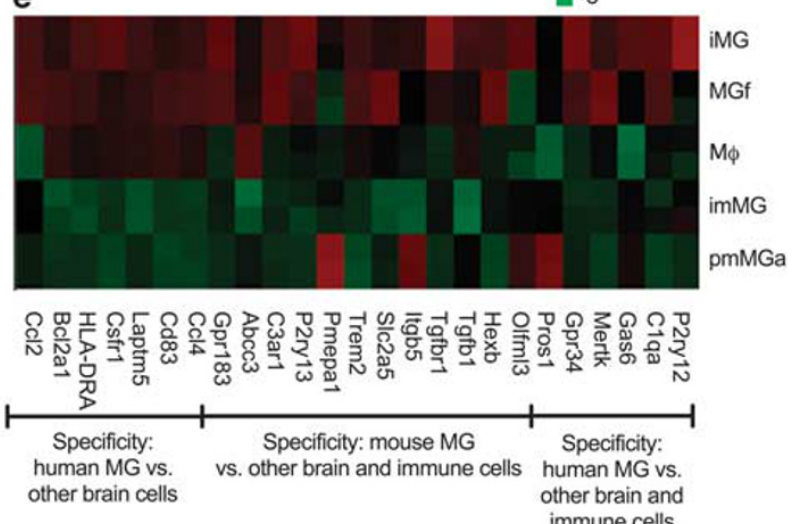

immune cells

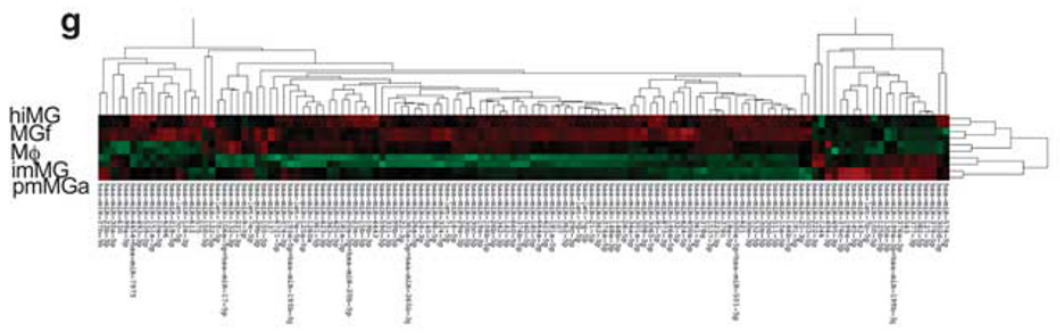

h

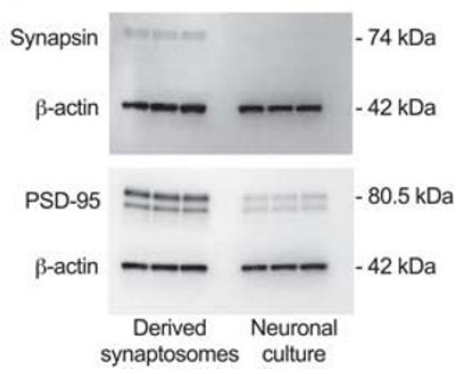

Figure 1. Characterizations of induced microglia-like cells (hiMG) and synaptosomes isolated from neural progenitor cell (NPC)-derived neural cultures. (a) Representative phase-contrast image of hiMG. (b-d) Immunocytochemistry of hiMG stained with antibodies to (b) P2RY12, (c) CX3CR1, (d) merged P2RY12 (red), CXCR1 (green) and nuclear Hoechst (blue). Scale bar $=60 \mu \mathrm{m}$. (e) Heat map based on hierarchical clustering of hiMG, monocyte-derived macrophages $(M \Phi)$, primary fetal microglia (MGf), SV40 immortalized primary microglia (imMG) and microglia isolated from adult post-mortem brain tissue (pmMGa). Results were log2-transformed, normalized to a set of housekeeping genes (ACTB, b2M, IDHA and PGK1), centered, and populations were clustered using an agglomerative algorithm. The data are presented as duplicates representative of two subjects for hiMG, $M \Phi, M G f$ and pmMGa, and one subject for imMG. (f) Hierarchical cluster analysis (median linking and correlation as distance metric) of the data in (e) using the R package pvclust. Uncertainty in clustering is assessed via multiscale bootstrap resampling ( $\mathrm{nboot}=10,000$ ) and Approximately Unbiased $(\mathrm{AU})$ probability value as well as ordinary Bootstrap Probability (BP) value is provided for each cluster. Clusters with a significance level of $<0.05$ corresponds to AU/BP $>95$, are highlighted. Standard error for $A U=99$ in the hiMG/MGf cluster was < 0.001). (g) Heat map and hierarchical clustering of human hiMG, M $\Phi$, MGf, imMG, pmMGa, using a Nanostringbased miRNA chip. Results were log2-transformed, normalized to the housekeeping gene RPLPO and centered, and populations were clustered using an agglomerative algorithm. The data are presented as duplicates representative of $n=2$ for hiMG, M $\Phi$, MGf, pmMGa and $n=1$ for imMG. See also Supplementary Figure S4 for analysis using pvclust. (h) Western blot of synapsin (presynaptic protein) and PSD-95 (postsynaptic protein) in synaptosomes and their corresponding NPC-derived neural cultures. Blot is representative for two subjects in triplicates.

\section{Confocal microscopy}

For confocal microscopy we used IN Cell 6000 analyzer (GE Healthcare, Chicago, IL, USA). Ten randomly selected images were acquired per well (24-well-plates). All settings were kept constant between different conditions. Quantification was performed using the Multi Target Analysis Module for IN Cell Analyzer 1000 (GE Healthcare). Nuclei were identified using a top-Hat transform, while cells were identified using region growing segmentation. pHrodo-labeled synaptosomes, PSD-95 or total C4 were identified using a multiscale top-Hat transform (10 transformations), a size filter of $0.5-1.5 \mu \mathrm{m}$, minimum sensitivity for detection of inclusions, and restriction of detection of inclusions to cell area. For outcome measurements we divided total detected area per image with total cell area per image.

\section{Statistics}

All cellular experiments were performed using cells from one or two subjects in triplicates and repeated twice. Group comparisons 

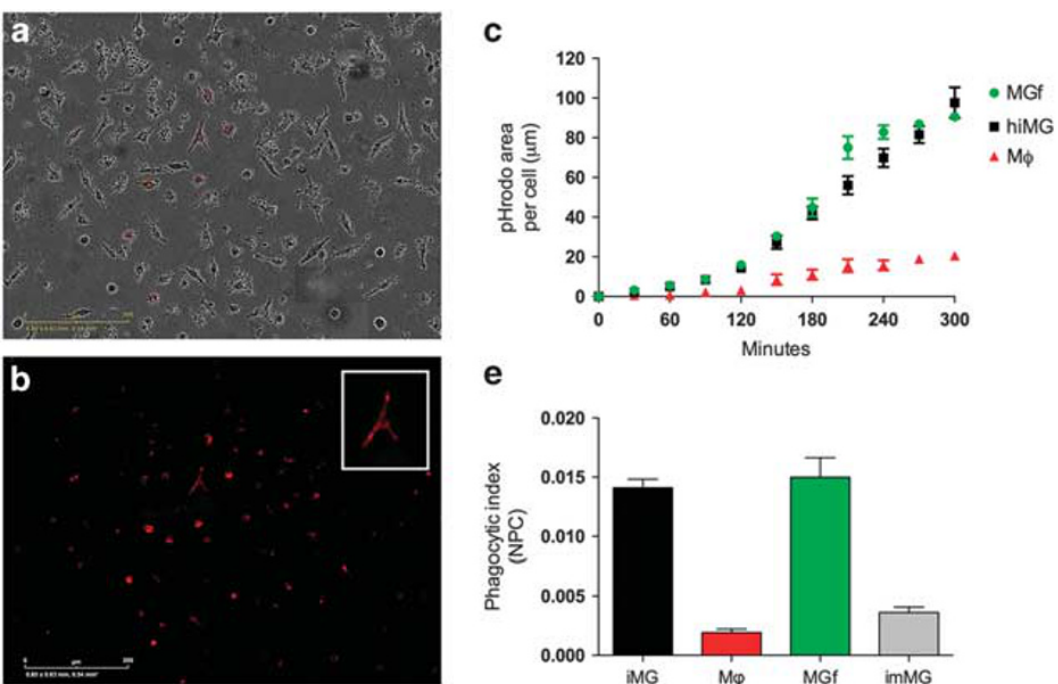

e
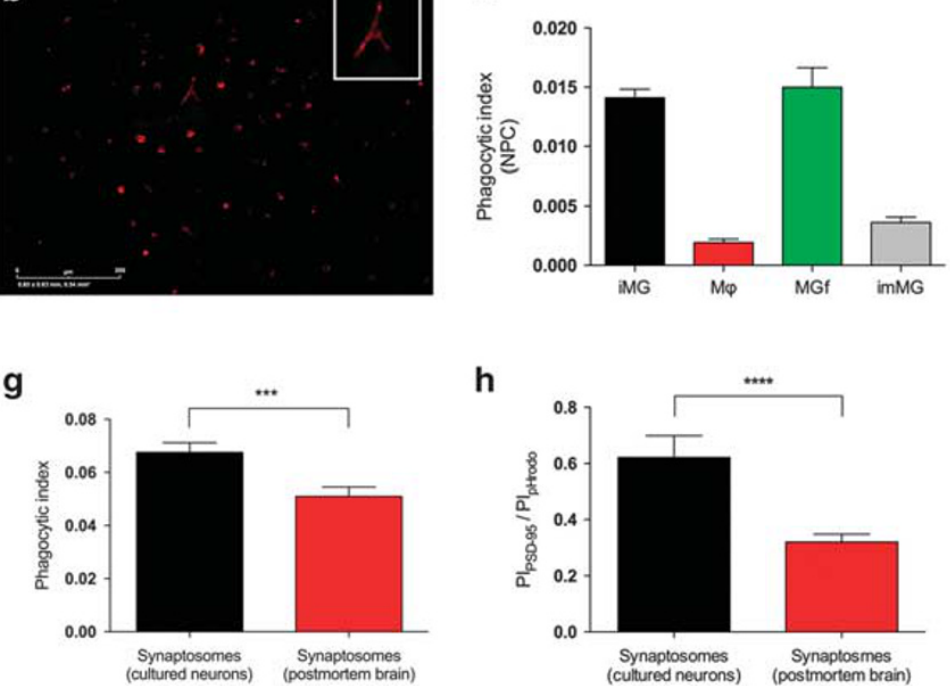

h

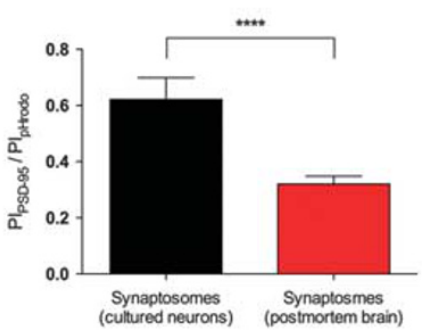

d

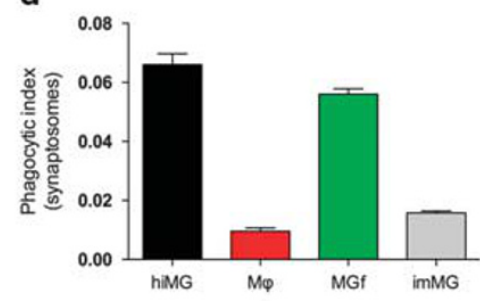

f

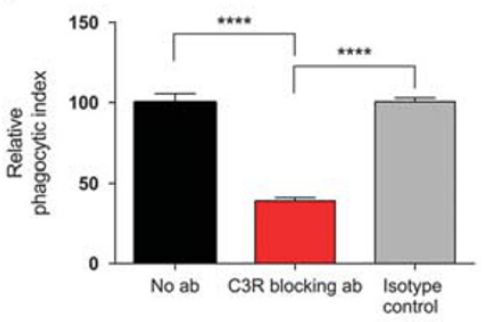

Figure 2. In vitro assays studying engulfment of synaptosomes and neural progenitor cells (NPCs) using human microglia-like cells (hiMG) from two subjects, human fetal primary microglia (MGf) from one subject, human monocyte-derived macrophages (M $\Phi$ ) from two subjects and a human SV40 immortalized microglia cell line (imMG). (a, b) Representative imaging real-time live images in phase-contrast/red fluorescence mode (a) and in red fluorescence mode (b) of pHrodo (red)-labeled synaptosomes uptake in hiMG after 5 h. (c) Quantification of pHrodo (red)labeled synaptosomes uptake (live imaging; 5 h). hiMG vs $M \Phi ; P<0.0001$, hiMG vs MGf; $P=N S$. (d) Quantification of pHrodo (red)-labeled synaptosomes uptake (confocal microscopy). hiMG vs M $\Phi ; P<0.001$, hiMG vs MGf; $P=$ NS. See Supplementary Figure S7 for representative confocal images. (e) Uptake in hiMG $(5 \mathrm{~h})$ of pHrodo (red)-labeled NPCs using confocal microscopy. hiMG vs $M \Phi ; P<0.0001$, hiMG vs MGf; $P=$ NS, hiMG vs imMG; $P=0.0001$. See Supplementary Figure S8 for representative confocal images. (f) Relative uptake of pHrodo (red)-labeled synaptosomes $(5 \mathrm{~h})$ in hiMG with and without $30 \mathrm{~min}$ of anti- $\alpha_{M}(C D 11 \mathrm{~b}) \mathrm{M} 1 / 70$ antibody pre-treatment as well as with pre-treatment using an isotype control antibody. Data are normalized to 'no antibody' (confocal microscopy). (g) hIMG uptake of pHrodo (red)-labeled synaptosomes isolated from NPC-derived neuronal cultures vs brain after $5 \mathrm{~h}$ (confocal microscopy; experiments controlled by total protein amount added). (h) Ratio of PSD-95 and phRodo immunoreactivity in hiMG treated with synaptosomes $(5 \mathrm{~h})$ isolated from NPC-derived neuronal cultures vs brain (confocal microscopy). (i, j) Representative confocal images of hiMG treated with cultured synaptosomes (i) and with synaptosomes isolated from brain (j). Scale bar $=60 \mu \mathrm{m}$. Error bars represent s.e.m. Phagocytic index represents total uptake area of puncta (0.5-1.5 $\mu \mathrm{m})$ located in cell area and divided on cell area. All $P$-values are two-sided. ${ }^{* * * *} P<0.01,{ }^{* * * * *} P<0.0001$.

were performed using Mann-Whitney U-test, Kruskal-Wallis $H$ test with Dunn's post hoc tests, or two-way repeated ANOVA (log-transformed values) with Tukey's post hoc tests using R statistics (R Development Core Team, Vienna, Austria). Graphs were produced in Graph-Pad prism 6.0 (http://www.graphpad. $\mathrm{com} /)$.

\section{RESULTS}

Generating and characterizing human microglia-like cells

First, we used PBMCs from cryopreserved healthy donor buffy coat preparations to generate human microglia-like cells (hiMG) using an induction supplement consisting of IL-34 (100 $\left.\mathrm{ng} \mathrm{ml}^{-1}\right)$ and granulocyte macrophage colony-stimulating factor $\left(10 \mathrm{ng} \mathrm{ml}^{-1}\right)$ as described previously for using fresh PBMCs. ${ }^{19}$ We here also utilized an extracellular matrix, Geltrex, resembling astrocytederived extracellular matrix (containing laminin, collagen and entactin) to obtain a robust, reproducible resting-state phenotype. After 2 weeks of serum-free culture conditions the majority of hiMG displayed a ramified morphology (Figure 1a), with positive immunostaining for the resident microglia surface marker P2RY12 (Figure 1b) as well as CX3CR1 (Figure 1c), TMEM119, IBA1, PU.1 and $\mathrm{CD} 11 \mathrm{~b}$ (Supplementary Figure S3). In order to further establish that hiMG displayed a microglia-specific signature, we constructed a Nanostring array based on genes reported to be unique or highly enriched in global gene expression analyses of primary microglia acutely isolated from patients undergoing nontumor brain surgery as well as in mouse. ${ }^{2,16}$ This gave us three sets of genes suggested to be highly enriched in (1) human microglia compared with other immune cells, (2) human or mouse microglia compared with other immune cells and (3) human microglia compared with other brain cells. For comparison we used monocyte-derived macrophages, primary microglia obtained from fetal brain tissue (cultured under serum-free conditions), primary microglia obtained from adult post-mortem brain tissue (serum starved for $24 \mathrm{~h}$ ) and an SV40 immortalized human microglia cell line (serum starved for $24 \mathrm{~h}$ ). Unsupervised hierarchical cluster analysis using all gene sets suggested that hiMG most closely resembled fetal primary microglia (Figure 1e). A cluster bootstrapping analysis $(10,000$ iterations) also confirmed strong 

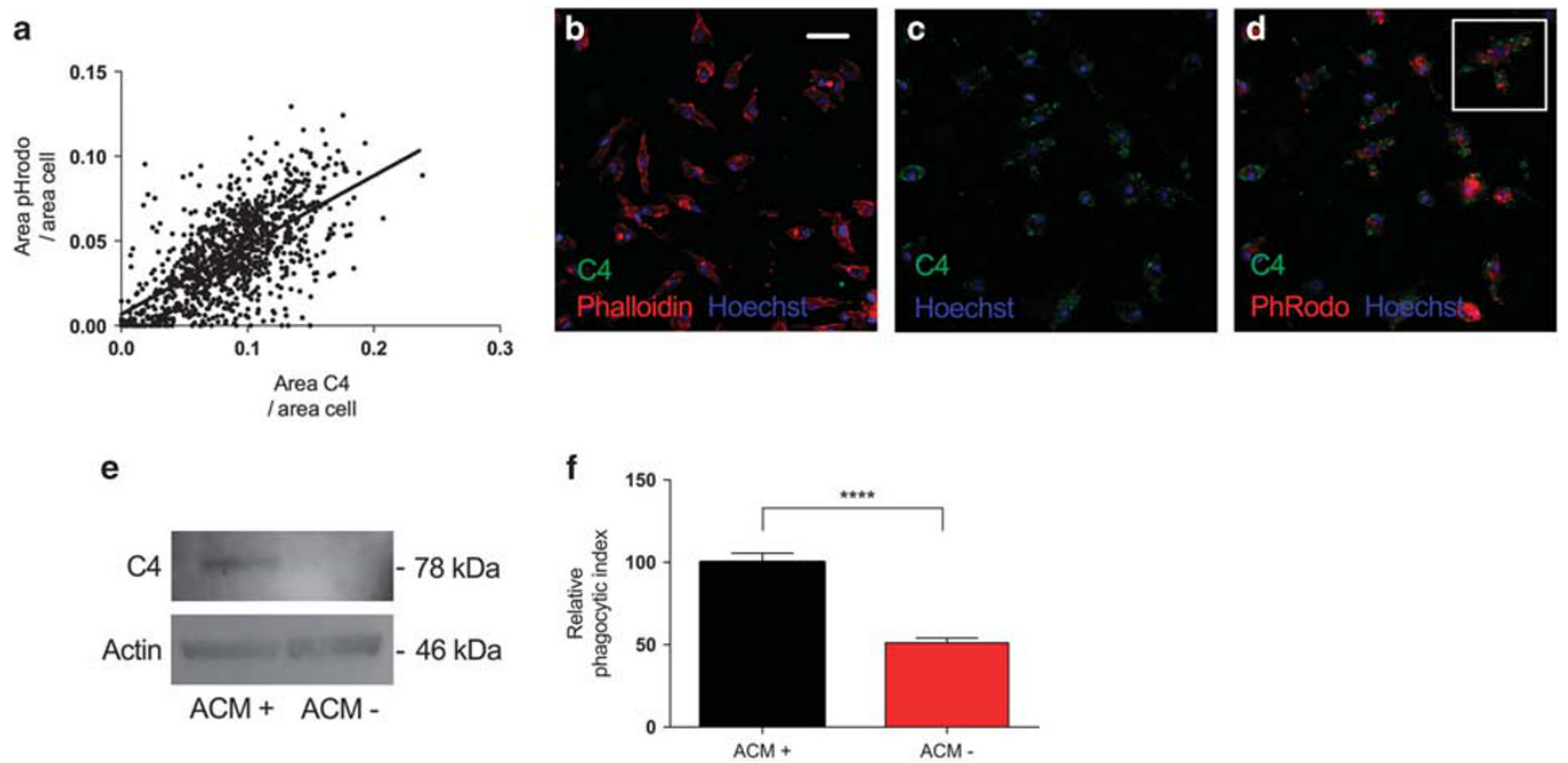

Figure 3. (a) Total area pHrodo+puncta in each cell plotted against total area C4+puncta in each cell (both measurements divided on cell area). $r=0.6(P<0.0001)$. Data were extracted from two randomly selected images with 999 cells in total. (b-d) Representative confocal images of C4+puncta in human induced microglia-like cells (hiMG) at baseline (b) and C4+puncta after treatment with synaptosomes (c, d). Quantification also confirmed increased C4 immunoreactivity post-assay $(P<0.0001)$. (e) Western blot analysis of C4 protein in NPC-derived neurons with and without pre-treatment with astrocyte-conditioned medium (ACM). Blot is representative of three experiments. (f) Relative uptake of pHrodo-labeled synaptosomes in hiMG with and without pre-treatment with ACM. Scale bar $=60 \mu \mathrm{m}$. Error bars represent s.e.m. Phagocytic index represents total uptake area of puncta $(0.5-1.5 \mu \mathrm{m})$ located in cell area and divided on cell area. All $P$-values are two-sided. $P<0.0001$.

statistical support for this cluster with an AU measure of 99 (s.e. $<0.001$ ) (Figure 1f). In comparison with macrophages that were suggested to form a separate cluster, hiMG displayed upregulation of 18 out of 20 genes selected primarily for enrichment in microglia vs other immune cells, including all genes highly or uniquely expressed in human microglia (P2ry12, C1qa, Gas6, Mertk, Gpr34 and Pros1). ${ }^{2}$ hiMG as well as primary fetal microglia also displayed a high relative expression of genes highly or uniquely enriched in human microglia vs other brain cells, while in agreement with previous data ${ }^{2}$ the expression in adult microglia from post-mortem tissue, as well as the immortalized cell line, displayed markedly lower expression throughout these two sets of genes. Notably, the use of post-mortem samples with shorter post-mortem interval (here, samples had a post-mortem interval of 9-12 h), other isolation methods, as well as culture conditions could possibly yield cells with greater expression of microglial-specific markers. In addition to mRNA expression, using a Nanostring-based miRNA chip containing probes specific for human 800 miRNAs, we again observed a very similar pattern with clustering of hiMG and fetal primary microglia (Figure $1 \mathrm{~g}$ and Supplementary Figure S4). Notably, further characterization of the transcriptome in comparison with other cell types (e.g., using RNA seq) may yield additional insights.

Generating human neural progenitor cells and isolated synaptic structures

For neural modeling we reprogrammed fibroblasts derived from skin punch biopsy into human iPSCs under xeno-free conditions. NPCs were derived from human iPSCs using a neural induction supplement based on defined small-molecule induction as described previously. ${ }^{20}$ NPCs were further purified to homogeneity using magnetic-activated cell sorting for PSA-NCAM+ cells verified by immunocytochemical analyses for standard NPC markers (PAX6, SOX1, SOX2 and NESTIN; data not shown) and a custom Nanostring array of NPC marker-specific gene expression (Supplementary Figure S5). We differentiated NPCs for 6 weeks into NPC-derived neural cultures for the purpose of isolating synaptosomes, that is, isolated nerve terminals. To account for astrocyte-derived factors, which in a previous report has been implicated in complement-dependent engulfment of synaptic terminals in mouse, ${ }^{21}$ we also exposed the neuronal cell cultures to ACM for $24 \mathrm{~h}$ before isolation of synaptosomes. NPC-derived neurons stained positive for MAP2, SMI312 and BIII-Tubulin (Supplementary Figure S6). Specificity of the synaptosome extraction procedure was confirmed by western blot analyses for the presynaptic marker synapsin as well as the postsynaptic marker PSD-95 (Figure 1h).

Complement-dependent synaptic pruning and elimination of neural progenitors in vitro

The ability of hiMG to engulf synaptosomes isolated from NPCderived neural cultures was first established using real-time live imaging. Synaptosomes were conjugated to a pH-sensitive dye (pHrodo) that enables quantification of synaptosomes localized to lysosomes. Quantification of bright-red fluorescence for $5 \mathrm{~h}$ revealed an efficient engulfment by hiMG as well as human fetal primary microglia (Figures 2a-c, Supplementary Figure S7 and Supplementary Movies S1 and S2). Conversely, the uptake of pHrodo-labeled synaptosomes in macrophages was markedly lower (Figure 2c). This was also in agremeent with our gene expression data displaying an upregulation in hiMG of genes critical for microglial phagocytosis. ${ }^{9,22-24}$ To obtain a more precise measurement of synaptosome uptake, we also quantified pHrodopositive puncta $(0.5-1.5 \mu \mathrm{m})$ inside cell bodies using confocal microscopy. The relative phagocytic capacity (total uptake area of puncta located in cell area and divided on cell area) displayed a similar pattern to our observations using live imaging (Figure $2 \mathrm{~d}$ ). Next, we studied engulfment of live NPCs using an identical assay. 
hiMG as well as primary microglia had the capacity to eliminate live NPCs while we observed sparse uptake with macrophages and a SV40 immortalized microglia cell line (Figure $2 \mathrm{e}$ and Supplementary Figure S8). To study the role of complement proteins in engulfment of synaptosomes by human microglia, we also repeated these experiments after pre-treating hiMG and human fetal primary microglia with an anti- $a_{M}$ (clone $M 1 / 70$ ) antibody in order to inhibit C3 receptor (C3R)-dependent phagocytosis by blocking the $a_{M}$ subunit (CD11b) of $C 3 R{ }^{25}$ This resulted in a greatly reduced pHrodo uptake in hiMG (Figure $2 f$ ) as well as in primary fetal microglia (data not shown). To further demonstrate the utility of patient NPC-derived neural synaptosomes, we compared these to synaptosomes obtained from postmortem brain biopsy. hiMG displayed a slightly greater phagocytic capacity for synaptosomes derived from culture (Figure $2 \mathrm{~g}$ ) and the proportion of PSD-95-positive puncta was substantially greater in cells exposed to synaptosomes from NPC-derived neuronal cultures (Figures $2 \mathrm{~h}-\mathrm{j}$ ). This suggests that isolating synaptosomes from in vitro neuronal cultures facilitates specific measurement of synaptic elimination.

C4 in modeling synaptic pruning using human cells

We applied the procedure to illustrate the application of this model system for disease-relevant mechanistic studies by investigating the role of $\mathrm{C} 4$ in human microglia-mediated pruning. Recently, reduced synaptic pruning in $\mathrm{C}^{-1-}$ mice was reported while altered C $4 A$ expression in human post-mortem brain was associated with schizophrenia risk. ${ }^{7}$ As schizophrenia patients display reduced numbers of synapses, ${ }^{26}$ excessive synapse elimination by microglia via a C4-dependent mechanism has been suggested in schizophrenia, but not directly examined in vitro. First, we examined total C4 levels in hiMG using immunocytochemistry. At baseline, hiMG as expected displayed sparse C4 immunoreactivity, but after $5 \mathrm{~h}$ of exposure of hiMG to synaptosomes we could observe strong and significantly increased C4 immunostaining (Figures $3 a-d$ ). The uptake of pHrodo-labeled synaptosomes and C4 immunoreactivity also correlated strongly on cell level (Figure 3a) and several C4positive puncta co-localized with pHrodo-labeled synaptosomes (Figure 3d). Thus, we reasoned that the observed increase in hiMG C4 immunoreactivity originated at least in part from C4-tagged synaptosomes. Western blot of isolated synaptosomes also revealed C4 protein (Supplementary Figure S9). Interestingly, and in agreement with data from mouse, ${ }^{21} 24 \mathrm{~h}$ exposure of neural cultures to ACM also increased total C4 levels (Figure 3e) and resulted in a doubling in uptake of pHrodo-labeled synaptosomes (Figure 3f). Importantly, a contribution of gliaderived C4 cannot be excluded and could be addressed in future studies by cell-specific C4 knockouts. Although more detailed mechanistic studies are needed, our results together with previous data from mouse suggest a role for C4 proteins in human microglia-mediated synaptic pruning, as well as the involvement of astrocyte-derived signaling in regulating human neural C4 expression.

\section{DISCUSSION}

In summary, we present and validate a novel model system using microglia-like cells and neural synaptosomes derived from living patient donors collected during a single brief clinical visit directly applicable to studying microglia-neural interactions towards understanding disease etiology. We demonstrate using this system and novel imaging assays that patient-derived reprogrammed microglia-like cells have the ability to engulf synaptosomes as well as NPCs in vitro. While surrogate models in rodents have the benefit of providing in vivo measurements, our model system provides opportunities for high-throughput drug screening using easily quantifiable assays. Further, this approach is also suitable for application to human disease association studies of complex disorders. The use of frozen blood samples and skin biopsies as the only necessary material from patients should facilitate well-powered studies that are unlikely to be feasible using post-mortem tissue alone.

\section{CONFLICT OF INTEREST}

CMS discloses lecture and consulting fees from Otsuka Pharmaceutical and $\mathrm{H}$ Lundbeck A/S (none of these are relevant to this work). RHP has served on scientific advisory boards or consulted to Genomind, Perfect Health, Psybrain, and RID Ventures (none of these are relevant to this work). The remaining authors declare no conflicts of interest.

\section{ACKNOWLEDGMENTS}

We are grateful to Jayla Ruiliera for technical assistance with isolating buffy coats. This work was supported by P50-MH106933 (NIMH and NHGRI), R01AT009144 $(\mathrm{NCClH})$ and Knut och Alice Wallenbergs Stiftelse.

\section{REFERENCES}

1 Aguzzi A, Barres BA, Bennett ML. Microglia: scapegoat, saboteur, or something else? Science 2013; 339: 156-161.

2 Butovsky O, Jedrychowski MP, Moore CS, Cialic R, Lanser AJ, Gabriely G et al. Identification of a unique TGF-beta-dependent molecular and functional signature in microglia. Nat Neurosci 2014; 17: 131-143.

3 Olah M, Raj D, Brouwer N, De Haas AH, Eggen BJ, Den Dunnen WF et al. An optimized protocol for the acute isolation of human microglia from autopsy brain samples. Glia 2012; 60: 96-111.

4 Schafer DP, Lehrman EK, Kautzman AG, Koyama R, Mardinly AR, Yamasaki R et al. Microglia sculpt postnatal neural circuits in an activity and complementdependent manner. Neuron 2012; 74: 691-705.

5 Stevens B, Allen NJ, Vazquez LE, Howell GR, Christopherson KS, Nouri N et al. The classical complement cascade mediates CNS synapse elimination. Cell 2007; 131: 1164-1178.

6 Paolicelli RC, Bolasco G, Pagani F, Maggi L, Scianni M, Panzanelli P et al. Synaptic pruning by microglia is necessary for normal brain development. Science 2011; 333: $1456-1458$.

7 Sekar A, Bialas AR, de Rivera H, Davis A, Hammond TR, Kamitaki N et al. Schizophrenia risk from complex variation of complement component 4 . Nature 2016; 530: 177-183.

8 Cunningham CL, Martinez-Cerdeno V, Noctor SC. Microglia regulate the number of neural precursor cells in the developing cerebral cortex. J Neurosci 2013; 33: 4216-4233.

9 Brown GC, Neher JJ. Microglial phagocytosis of live neurons. Nat Rev Neurosci 2014; 15: 209-216.

10 Derecki NC, Cronk JC, Lu Z, Xu E, Abbott SB, Guyenet PG et al. Wild-type microglia arrest pathology in a mouse model of Rett syndrome. Nature 2012; 484: 105-109.

11 Hong S, Beja-Glasser VF, Nfonoyim BM, Frouin A, Li S, Ramakrishnan S et al. Complement and microglia mediate early synapse loss in Alzheimer mouse models. Science 2016; 352: 712-716.

12 Lui H, Zhang J, Makinson SR, Cahill MK, Kelley KW, Huang HY et al. Progranulin deficiency promotes circuit-specific synaptic pruning by microglia via complement activation. Cell 2016; 165: 921-935.

13 Smith AM, Dragunow M. The human side of microglia. Trends Neurosci 2014; 37: 125-135.

14 Chung WS, Clarke LE, Wang GX, Stafford BK, Sher A, Chakraborty C et al. Astrocytes mediate synapse elimination through MEGF10 and MERTK pathways. Nature 2013; 504: 394-400.

15 Miksa M, Komura H, Wu R, Shah KG, Wang P. A novel method to determine the engulfment of apoptotic cells by macrophages using pHrodo succinimidyl ester. J Immunol Methods 2009; 15342: 71-77.

16 Darmanis S, Sloan SA, Zhang Y, Enge M, Caneda C, Shuer LM et al. A survey of human brain transcriptome diversity at the single cell level. Proc Natl Acad Sci USA 2015; 112: 7285-7290.

17 Suzuki R, Shimodaira H. Pvclust: an R package for assessing the uncertainty in hierarchical clustering. Bioinformatics 2006; 22: 1540-1542.

18 Ribes S, Ebert S, Czesnik D, Regen T, Zeug A, Bukowski S et al. Toll-like receptor prestimulation increases phagocytosis of Escherichia coli DH5alpha and Escherichia coli K1 strains by murine microglial cells. Infect Immun 2009; 77: 557-564. 
19 Ohgidani M, Kato TA, Setoyama D, Sagata N, Hashimoto R, Shigenobu K et al. Direct induction of ramified microglia-like cells from human monocytes: dynamic microglial dysfunction in Nasu-Hakola disease. Sci Rep 2014; 4: 4957.

20 Li W, Sun W, Zhang Y, Wei W, Ambasudhan R, Xia P et al. Rapid induction and long-term self-renewal of primitive neural precursors from human embryonic stem cells by small molecule inhibitors. Proc Natl Acad Sci USA 2011; 108: 8299-8304.

21 Bialas AR, Stevens B. TGF-beta signaling regulates neuronal C1q expression and developmental synaptic refinement. Nat Neurosci 2013; 16: 1773-1782.

22 Fourgeaud L, Traves PG, Tufail Y, Leal-Bailey H, Lew ED, Burrola PG et al. TAM receptors regulate multiple features of microglial physiology. Nature 2016; 532: 240-244.

23 Preissler J, Grosche A, Lede V, Le Duc D, Krügel K, Matyash V et al. Altered microglial phagocytosis in GPR34-deficient mice. Glia 2015; 63: 206-215.

24 Kawabori M, Kacimi R, KauppinenT, Calosing C, Kim JY, Hsieh CL et al. Triggering receptor expressed on myeloid cells 2 (TREM2) deficiency attenuates phagocytic activities of microglia and exacerbates ischemic damage in experimental stroke. J Neurosci 2015; 35: 3384-3396.
25 Reichert F, Rotshenker S. Complement-receptor-3 and scavenger-receptor-AI/II mediated myelin phagocytosis in microglia and macrophages. Neurobiol Dis 2003; 12: $65-72$.

26 Glausier JR, Lewis DA. Dendritic spine pathology in schizophrenia. Neuroscience 2013; 251: 90-107.

(c) (i) (2) This work is licensed under a Creative Commons Attributioncc) NonCommercial-ShareAlike 4.0 International License. The images or other third party material in this article are included in the article's Creative Commons license, unless indicated otherwise in the credit line; if the material is not included under the Creative Commons license, users will need to obtain permission from the license holder to reproduce the material. To view a copy of this license, visit http:// creativecommons.org/licenses/by-nc-sa/4.0/

(c) The Author(s) 2017

Supplementary Information accompanies the paper on the Molecular Psychiatry website (http://www.nature.com/mp) 